\title{
P02-98
}

\section{MONITORING OF THE METABOLIC SYNDROME IN PSYCHIATRIC INPATIENTS}

J. Cordes ${ }^{1}$, G. Regenbrecht ${ }^{1}$, M.W. Agelink², J. Zielasek¹, K.G. Kahl ${ }^{3}$

${ }^{1}$ Heinrich-Heine-University Duesseldorf, Düsseldorf, ${ }^{2}$ Department of Psychiatry, Psychotherapy and Psychosomatic, Herford, ${ }^{3}$ University of Dresden, Dresden, Germany

In this naturalistic observational study carried out in an inpatient treatment setting we as yet surveyed the parameters of the metabolic syndrome. A weekly monitoring procedure was implemented. The analysis included data of 350 patients over a time of 12 weeks. The last observation carried forward method was applied. Additionally we are evaluating the informative value of visceral body fat percentage as measured by a body composition analyzer. The patients showed a weight increase over the first 12 weeks (mean increase: $0.87 \mathrm{~kg}, \mathrm{p}<.001$ ) as well as an increase of the body mass index (mean increase: $0.45 \mathrm{~kg} / \mathrm{m}^{2}, \mathrm{p}<.001$ ). Accordingly, waist circumference (mean increase: $1.06 \mathrm{~cm}, \mathrm{p}=.007$ ) and visceral fat index (mean increase: $0.19, p=.007$ ) increased. No worsening of fasting glucose and blood lipid concentrations was detected. Spearmens coefficient indicated correlations between visceral fat index and body mass index $(\rho=.77 ; p<.001)$, waist circumference $(\rho=.70 ; p<.001)$, and triglyceride concentrations $(\rho=.39 ; p<.001)$. Correlations between visceral fat index and fasting glucose $(\rho=.18 ; p=.019)$, and visceral fat index and total cholesterol $(\rho=.16 ; p=.049)$ were weak but also significant. In contrast, the HDL cholesterol showed a negative relation with $\rho<-.39$ at each point in time $(p<.001)$.

We conclude that psychiatric patients are at increased risk for the development of metabolic alterations during inpatient treatment. The possible underlying mechanisms of this interaction are discussed. 